\title{
Applying participatory design with dementia stakeholders: Challenges and lessons learned from two projects
}

\author{
Luiz Henrique Cavalcanti \\ School of Informatics and Computing- \\ IUPUI \\ Indiana, US \\ luccaval@iupui.edu
}

\author{
Yamini Karanam \\ School of Informatics and Computing- \\ IUPUI \\ Indiana, US \\ ykaranam@iupui.edu
}

\author{
Richard J Holden \\ Indiana University School of Medicine \\ Indiana, US \\ rjholden@iu.edu
}

\begin{abstract}
Researchers and designers find it challenging to use traditional methods to establish cognitive and affective empathy with persons with dementia (PWD) and informal caregivers of PWD (CPWD). We used participatory design (PD) to learn about and design for the experiences of PWD and CPWD. Here, we present our experience applying PD in a design project with PWD and another with CPWD. We discuss challenges we faced regarding choice of method, relationship with project partners, and working with an older, cognitively impaired population. Finally, we describe lessons learned using PD methods with PWD and CPWD.
\end{abstract}

\section{CCS CONCEPTS}

- Human-centered computing $\rightarrow$ Participatory design; Field studies $\bullet$ Applied computing $\rightarrow$ Consumer health

\section{KEYWORDS}

H.5 - Information interfaces and presentation (HCI); H.5.0 General.

\section{INTRODUCTION}

Dementia is a general term for the loss of mental abilities caused by degenerative disorders affecting the brain [1]. Designing for persons with dementia (PWD) and informal caregivers of PWD (CPWD) requires empathy from designers, but achieving empathy is difficult due to the unique cognitive, psychiatric, physical, and linguistic impairments of PWD [2-4]. Thus, directly interacting with PWD and CPWD is essential [5]. Participatory design (PD) methods in particular provide not only direct interaction but also opportunities for PWD and CPWD to add their voice to the design process [6]. However, implementing PD methods is challenging because of aforementioned impairment and other limitations [6,7].

In this paper we add to the nascent literature describing implementing PD with PWD and CPWD [8], as well as studies in general describing implementation challenges and strategies related to field research in the health domain $[9,10]$.

\section{CASE STUDY}

We conducted two projects as part of an academiccommunity partnership called B-PHIT Indy (BrainProtective Health Information Technology, Indianapolis). B-PHIT Indy was an action research initiative between the Health Innovation Lab at Indiana University - Indianapolis (USA) and local entities concerned with senior brain health, including two partners serving PWD and CPWD. The goals of the B-PHIT Indy initiative were to: 1) engage partnering community organizations in developing joint projects and 2) include "end-users" from the community in product co-design and evaluation. A key objective of the initiative was to uncover the challenges and lessons learned when performing PD with cognitively impaired individuals and their formal and informal caregivers.

\subsection{PD Approach}

In addition to learning how best to work with PWD and CPWD in a participatory manner, achieving project goals involved quickly resolving emerging research challenges and incorporating lessons learned in subsequent phases of PD. Thus, we modified a Design Thinking framework 
$[11,12]$ to incorporate multiple iterations of participant interactions within as well as between research phases. For example, in one project this meant multiple instances of PWD using a designed product, with small experimental changes to instructions and facilitation style during each session. These planned iterations afforded the team opportunities to quickly review and adjust methods $[13,14]$.

In addition, we adopted the agile concept of featurebased design, where the design product is divided into features that can be designed and tested independently $[15,16]$, supporting short-term deliverables while concurrently managing multiple features.

\subsection{Project 1: CARE Kit}

The CARE Kit project's goals were to: 1) investigate the current use of a paper-based information management system called the CARE Kit and 2) provide design recommendations for a digital version of the CARE Kit. The paper CARE Kit enabled caregivers to assemble, share, update, and manage care recipients' information. However, the community partner and academic research team shared a vision of leveraging digital tools to facilitate better aggregation, updating, portability, security, and sharing of personal health information better matched to the dynamic needs of caregivers, particularly in light of the progressive nature of dementia in aging individuals $[17,18]$.

The project used narrative interviews [19] and card sorting [12] with CPWD and paid staff at Joy's House, an Indianapolis (USA) based not-for-profit adult day service. We interviewed ten caregivers and four day-service staff about their caregiving experiences, information management practices, and use of CARE Kit. We performed card sorting and artifact analysis to understand caregivers' use of and prioritization of care recipients' personal health information.

\subsection{Project 2: Fulfilling activities}

This project aimed to create an intervention to engage PWD in enjoyable and rewarding meaningful activities. The project's two phases were done with community partners Joy's House and the Aging Brain Care outpatient program of a large community medical center.

In phase one, we performed six group interviews with professionals: three sessions with Joy's House staff and volunteers and three with Aging Brain Care nurses and social workers. Participants (usually 3-5 per session) were prompted to discuss activity content, delivery strategies, and outcomes for PWD engaging in meaningful activities.
In parallel, the team engaged in weekly participantobserver immersions as activity volunteers at Joy`s House, engaging with PWD with different levels of impairment.

Phase two was comprised of PD sessions, prototyping, and testing interfaces of iterative prototypes of "Joy in a Box", a portable activity kit offering PWD safe activities tailored to different levels of impairment, identity, and interests. We tested individual engagement with prototypes and specific hypotheses about design components (e.g., placement of instructions, physical product design).

\section{CHALLENGES}

During the execution of the case projects, we faced a series of challenges to performing PD activities with the participant population of PWD and CPWD.

\section{Impairment related challenges}

Involving PWDs in PD activities resulted in logistic challenges such as slow-paced recruitment, in line with prior findings [20-22]. We also faced challenges related to participants' capacity to comprehend and perform the design methods. For instance, during the first PD session for "Joy in a Box", participants engaged with the prototype of a gardening kit and discussed it afterwards. However, it was challenging to communicate the goal of the activity and interpret participant comments without providing guidance on using the prototype itself.

Another impairment-related challenge was the tendency to exhibit mood swings and rapid changes of opinion. This required having additional material and, when possible, a backup plan for such cases. To perform group activities, the team had to become familiar with specific behavioral patterns of participants: for instance, Mr. Thomas likes to hug and Ms. Devlin does not like to talk but loves to draw. The team also had to understand how participants functioned as a group, how their personalities impacted each other, and how group dynamics affected the activity.

\section{Relationship challenges}

As enriching as it was to include stakeholders with different backgrounds and expertise from multiple community organizations, communicating the expectations and outcomes of each research activity was challenging.

Additionally, the capacity to establish a comfortable space and trust with participants (both PWD and CPWD) proved crucial to achieving success. Participating in narrative interviews led some CPWD to recall emotional and stressful memories. Moderating emotionally stressful sessions required our personnel to learn to be neutral and 
task-focused on the one hand and empathetic and comforting on the other.

\section{Methodological challenges}

With multiple methods being used simultaneously, the team had to manage several procedure-specific challenges.

For instance, during project 2, we had to organize the team's work schedule to accommodate parallel discussions with two different groups of participants and summarize the findings before the next round of interviews. We ended up with a weekly schedule cycling between group interviews, intensive team meetings for discussion and planning, and immersions. These activities informed each other and were scheduled to allow for efficient data collection with busy professionals.

\section{LESSONS LEARNED}

The research team also learned a variety of lessons, translating in six recommendations for future PD work of this type. The recommendations complement others related to ensuring safety, methods quality and engagement when using PD methods with PWD [8, 23].

\section{Get to know participants individually}

During our procedures, we found that is important to have as much information as possible about each participant. This means knowing who they are, where they come from, what they like, and what they dislike. Knowing this will help the researcher not only make the participant more comfortable to engage in research activities but helps to anticipate and prepare for potential issues that can impact the study procedures. It is possible to collect information about participants by communicating with them in advance. Another possibility is to work with CPWD to understand individual PWD participants and collect advice on how to handle behavioral incidents. Early engagement with participants through immersion gave us a chance to acquire information about them, facilitating later project stages.

\section{Adjust methods to participant limitations and motivations}

Researchers should be able to adjust methods and materials to suit participant needs. Adjustments that worked included always having paper and colored pencils, budgeting extra time for sessions, splitting activities into multiple small sessions instead of one long session, and reserving additional space and materials for informal caregivers.

Much of the literature reporting guidelines and recommendations for $\mathrm{PD}$ with $\mathrm{PWD}$ focuses on preparing support materials and communicating with participants $[8,23]$. Additionally, we identified the importance of preparing the space to be used for the activity. Suggestions include clearing exterior elements that can distract or interfere with participants' attention, while at the same time providing comfort and familiarity to motivate engagement.

\section{Be respectful and open to change}

Researchers working with PWD and CPWD should always be open and attentive to participant requests, even ones perceived as impolite or unexpected. During a PD session, the researcher may need to identify underlying needs and adjust accordingly. If the activity is frustrating to a participant due to motor impairment, the researcher could shift to a conversational procedure, where the participant verbally guides task execution while the researcher performs the physical actions.

Have a plan $B$

In addition to adjusting methods within session, a secondary plan can be useful in case one method fails to collect desired data. For example, if group PD sessions did not work, the researcher may attempt individual sessions. If structured surveys failed, unstructured or semi-structured interviews may be indicated.

Gain the support of informal or formal caregivers Others have noted the important role CPWD can play in facilitating communication with PWD in PD activities [5], but the support of people close to the PWD can help both before and during PD procedures: to know more about the participant and to manage any issues that arise later. CPWD need not be an active part of the procedure; it may be sufficient that they are simply present.

\section{CONCLUSIONS}

Using PD methods with PWD and CPWD presents unique challenges compared to PD with unimpaired or differently impaired individuals. While ours was not an exhaustive list of all the challenges - and joys - that this entails, we urge designers and PD practitioners to plan for the ones we identified above as well as contribute their own.

For both projects in the B-PHIT Indy initiative, partnering with community organizations helped to anticipate and overcome many challenges, while offering opportunities to design and maximize results. By sharing our experiences and lessons learned we seek to both enlighten and engage our colleagues in: 1) using PD with PWD and CPWD; 2) describing their own experiences and strategies; and 3) performing investigations to rigorously test strategies towards an evidence base for PD practice in this important domain. 


\section{ACKNOWLEDGMENTS}

This work was supported by the Charles R. Bantz Community Scholar Award to RJH from the Office of the Vice Chancellor for Research of Indiana University Purdue University, Indianapolis. Thank you to the B-PHIT Indy community partners, research team, and participants.

\section{REFERENCES}

[1] Alzheimer's Association. (2017). 2017 Alzheimer's disease facts and figures. Alzheimer's \& Dementia, 13(4), 325-373.

[2] Barry Reisberg, Steven H. Ferris, Mony J. de Leon, and Thomas Crook. 1982. The Global Deterioration Scale for assessment of primary degenerative dementia. The American Journal of Psychiatry 139, 9: 1136-1139.

[3] G. M. Francis and B. A. Munjas. 1992. Needs of family caregivers and persons with Alzheimer's disease. American Journal of Alzheimer's Care and Related Disorders \& Research 7, 4: 23-31.

[4] Sarah B Wackerbarth and Mitzi M. S Johnson. 2002. Essential information and support needs of family caregivers. Patient Education and Counseling 47, 2: 95-100.

[5] Lindsay, S., Brittain, K., Jackson, D., Ladha, C., Ladha, K., \& Olivier, P. (2012, May). Empathy, participatory design and people with dementia. In Proceedings of the SIGCHI Conference on Human Factors in Computing Systems (pp. 521-530). ACM.

[6] Qaurooni, D., Ghazinejad, A., Kouper, I. and Ekbia, H., 2016, May. Citizens for science and science for citizens: The view from participatory design. In Proceedings of the 2016 CHI Conference on Human Factors in Computing Systems (pp. 1822-26). ACM.

[7] Hendriks, N., Huybrechts, L., Wilkinson, A., \& Slegers, K. (2014, October). Challenges in doing participatory design with people with dementia. In Proceedings of the $13^{\text {th }}$ Participatory Design Conference: Short Papers, Industry Cases, Workshop Descriptions, Doctoral Consortium papers, and Keynote abstracts-Volume 2 (pp. 33-36). ACM.

[8] Allen, R. S., Cook, S. E., Hignett, S. M., \& Jais, C. L. (2017). Involving people with dementia in participatory design: ethical processes and consent for dementia research. Alzheimer's \& Dementia: The Journal of the Alzheimer's Association, 13(7), P156-P157.

[9] Holden, R. J., McDougald Scott, A. M., Hoonakker, P. L. T., Hundt, A. S., \& Carayon, P. (2015). Data collection challenges in community settings: Insights from two field studies of patients with chronic disease. Quality of Life Research, 24(5), 1043-1055.

[10] Valdez, R. S., \& Holden, R. J. (2016). Health care human factors/ergonomics fieldwork in home and community settings. Ergonomics in Design, 24, 44-49.

[11] Beckman, S.L. and Barry, M., 2007. Innovation as a learning process: Embedding design thinking. California management review, 50(1), pp.25-56.

[12] Brown, T., \& Katz, B. (2009). Change by design: How design thinking transforms organizations and inspires innovation.

[13] Cockton, G., Lárusdóttir, M., Gregory, P., \& Cajander, Å. (2016). Integrating User-Centred Design in Agile Development. In Integrating User-Centred Design in Agile Development (pp. 1-46). Springer International Publishing.

[14] Singh, M., 2008, August. U-SCRUM: An agile methodology for promoting usability. In Agile, 2008. AGILE'08. Conference (pp. 555-560). IEEE.

[15] Li, W.D., Ong, S.K., Fuh, J.Y., Wong, Y.S., Lu, Y.Q. and Nee, A.Y., 2004. Feature-based design in a distributed and collaborative environment. Computer-Aided Design, 36(9), pp.775-797.

[16] Da Silva, T. S., Martin, A., Maurer, F., \& Silveira, M. (2011, August). User-centered design and agile methods: a systematic review. In Agile Conference (AGILE), 2011 (pp. 77-86). IEEE.

[17] Karla T. Washington, Susan E. Meadows, Susan G. Elliott, and Richelle J. Koopman. 2011. Information needs of informal caregivers of older adults with chronic health conditions. Patient Education and Counseling 83, 1: 37-44.

[18] R. Agarwal, J. Khuntia, Personal Health Information and the Design of Consumer Health Information Technology: Background Report. | AHRQ National Resource Center; Health Information Technology: Best Practices Transforming Quality, Safety, and Efficiency, Agency for Healthcare

[19] Sandra Jovchelovitch and Martin W. Bauer. 2000. Narrative interviewing. Qualitative researching with text, image and sound: $57-74$.

[20] Lindsay, S., Jackson, D., Schofield, G., \& Olivier, P. (2012, May). Engaging older people using participatory design. In Proceedings of the SIGCHI Conference on Human Factors in Computing Systems (pp. 1199-1208). ACM.

[21] Hendriks, N., Truyen, F., \& Duval, E. (2013, September). Designing with dementia: Guidelines for participatory design together with persons with dementia. In IFIP Conference on Human-Computer Interaction (pp. 649-666). Springer, Berlin, Heidelberg.

[22] Hendriks, N., Slegers, K., \& Wilkinson, A. (2017). Lessons Learned from Participatory Design in Dementia Care: Placing Care Partners at the Centre. Studies in health technology and informatics, 233, 63-77.

[23] Mayer, J. M., \& Zach, J. (2013, August). Lessons learned from participatory design with and for people with dementia. In Proceedings of the 15th international conference on Human-computer interaction with mobile devices and services (pp. 540-545). ACM. 